\title{
INFLUÊNCIA DA GRANULOMETRIA E DA MINERALOGIA SOBRE A RETENÇÃO DO FÓSFORO EM LATOSSOLOS SOB PASTAGENS NO CERRADO ${ }^{(1)}$
}

\author{
Diogo Néia Eberhardt ${ }^{(2)}$, Pedro Rodolfo Siqueira Vendrame ${ }^{(3)}$, \\ Thierry Becquer ${ }^{(4)}$ \& Maria de Fátima Guimarães ${ }^{(3)}$
}

\begin{abstract}
RESUMO
As pastagens abrangem cerca de 50 milhões de hectares na região do Cerrado. Os Latossolos, ocupando aproximadamente a metade da área, são solos intemperizados, constituídos, principalmente de caulinita, óxidos de Fe e $\mathrm{Al}$ e estes óxidos têm grande influência na retenção de $\mathrm{P}$. O objetivo deste trabalho foi avaliar os teores totais de $P$, assim como sua disponibilidade e retenção em Latossolos sob pastagens do Cerrado, e relacionar estes dados com suas características mineralógicas, granulométricas e químicas. As relações entre as características estudadas foram avaliadas por meio da análise de componentes principais e correlações de Pearson. O P disponível resina mostrou-se correlacionado negativamente com as características mineralógicas e granulométricas e positivamente com os teores de matéria orgânica do solo. Tanto os óxidos livres totais como os amorfos tiveram correlações negativas com o $\mathbf{P}$ remanescente (Prem). Os teores de gibbsita, goethita e hematita apresentaram correlações negativas com o Prem. Por outro lado, a caulinita não exerceu influência sobre o Prem, indicando a baixa participação desta na adsorção de $\mathbf{P}$ nos Latossolos do Cerrado. Observou-se correlação negativa entre o Prem e o C total, explicada pelo fato de o estoque de $\mathrm{C}$ aumentar linearmente com o conteúdo de argila e silte no solo. Os óxidos de $\mathrm{Fe}$ e $\mathrm{Al}$ foram os componentes mineralógicos que exerceram maior influência sobre o Prem.
\end{abstract}

Termos de indexação: disponibilidade de $P$, sorção de $P$, fósforo remanescente, óxidos de Fe e Al.

\footnotetext{
(1) Recebido para publicação em maio de 2007 e aprovado em março de 2008.

(2) Pós-Graduando do Departamento de Agronomia, Universidade Estadual de Londrina - UEL. Caixa Postal 6001, CEP 86051970 Londrina (PR). E-mail: diogone@yahoo.com.br

(3) Professor do Departamento de Agronomia, UEL. E-mail: mfatima@uel.br, perovendrame@yahoo.com.br

(4) Pesquisador do IRD, UMR 137 BioSol, Universités Paris VI et XII, Paris. E-mails: thierry.becquer@ird.fr
} 


\title{
SUMMARY: INFLUENCE OF SOIL TEXTURE AND MINERALOGY ON PHOSPHORUS RETENTION IN CERRADO OXISOLS UNDER PASTURE
}

\begin{abstract}
Pastures cover nearly 50 million hectares in the Cerrado region. The Oxisols, which cover approximately half this area, are highly weathered soils composed mainly of kaolinite, iron and aluminum oxides. Iron and aluminum oxides have a great influence on the retention of phosphorus $(P)$ in these soils. The aim of this study was to quantify the total phosphorus levels, as well as $P$ availability and retention in Oxisols under pasture in the Cerrado region and to relate the data with soil mineralogical, textural and chemical characteristics. The relations between the studied parameters were analyzed by principal component analysis and linear correlation analysis. The ion-exchange resin extractable $P$ was negatively correlated with mineralogical and texture soil characteristics, and positively with soil organic matter contents. Both the total free and the poorly crystalline oxides were negatively correlated with equilibrium $P$ (Prem). Gibbsite, goethite as well as the total free and poorly crystalline oxides were negatively correlated with Prem, indicating their effects on $P$ adsorption to Cerrado Oxisols. Conversely, kaolinite was not significantly correlated with Prem. The negative correlation between Prem and total carbon ( $C$ total) was due to the linear increase of soil carbon with the clay and silt contents. The iron and aluminum oxides were the characteristics that most influenced Prem of Cerrado Oxisols.
\end{abstract}

Index terms: Oxisols, $P$ availability, $P$ sorption, remaining phosphorus, $F$ e and Al oxides.

\section{INTRODUÇÃO}

A região do Cerrado, segundo maior bioma do Brasil, ocupa uma área de $204 \mathrm{M}$ ha (Souza \& Lobato, 2004). As superfícies de pastagens nativas e cultivadas são estimadas em $117 \mathrm{M}$ ha, das quais, segundo Sano et al. (2000), 49,5 M ha são de pastagens cultivadas. O sistema extrativista característico desta atividade pode ser confirmado pela irrisória quantia de $4 \mathrm{~kg} \mathrm{ha}^{-1} \mathrm{de}$ NPK que as pastagens brasileiras receberam, em média, no período de 1994 a 2002 (Vilela et al., 2004). Este dado reforça as estimativas de Brossard \& Barcelos, (2005) de que entre 50 e 80 \% das pastagens do Cerrado apresentam algum grau de degradação.

Os Latossolos representam aproximadamente $45,7 \%$ dos solos da região do Cerrado, sendo descritos como muito intemperizados, profundos, não-hidromórficos, com teor de argila entre 15 e $80 \%$ e pequena reserva de nutrientes para as plantas. Mais de $95 \%$ são distróficos ou álicos e apresentam $\mathrm{pH}$ entre 4,0 e 5,5, baixa capacidade de troca catiônica, alta capacidade de adsorção aniônica (especialmente fosfato) e teores baixos de $\mathrm{P}$ disponível por Mehlich-1 (menores que 1,0 mg kg-1) (Resende et al., 1995).

A fração argila destes Latossolos é composta principalmente por caulinita, óxidos de $\mathrm{Fe}$ (goethita e hematita) e óxidos de $\mathrm{Al}$ (gibbsita). Alguns Latossolos, formados de rochas mais ricas em $\mathrm{Fe}$, apresentam, na fração argila, a maghemita e, na fração areia, a magnetita e a ilmenita (Souza \& Lobato, 2004). Bahia Filho (1982) testou o efeito dos componentes mineralógicos da fração argila sobre a capacidade máxima de adsorção de $\mathrm{P}$ (CMAP) e verificou que a goethita $(\mathrm{Gt})$ foi a principal responsável, contribuindo com 86 \% do total da CMAP. Segundo Borggaard (1983) e Torrent et al. (1994) os solos goethíticos, geralmente, fixam mais $\mathrm{P}$ do que os hematíticos, por ser menor a superfície específica da hematita, decorrente da maior dimensão dos cristais, o que desfavorece a fixação de $\mathrm{P}$. Os óxidos de Fe amorfos, apesar de serem quantitativamente uma fração secundária, podem influenciar a sorção do P. De acordo com Borggaard (1983), os óxidos de Fe amorfos adsorvem 3,5 vezes mais $\mathrm{P}$ que os óxidos de Fe bem cristalizados, por apresentarem maior superfície específica (2,9 vezes).

Dubus \& Becquer (2001) verificaram correlação negativa entre a sorção de $\mathrm{P}$ e o teor de $\mathrm{C}$ orgânico. Para Afif et al. (1995), esta observação parece estar relacionada com a ação bloqueadora da matéria orgânica sobre os sítios de adsorção. Considerando que tal efeito possa ser temporário, fica evidente que a manutenção de teores altos de matéria orgânica (MO) é imprescindível para amenizar os efeitos da adsorção de $\mathrm{P}$ nos solos.

A variabilidade da fração argila em Latossolos é amplamente conhecida (Bahia Filho, 1982; Fontes \& Weed, 1996; Alves \& Lavorenti, 2004). Por outro lado, a contribuição de seus componentes na sorção de $\mathrm{P}$ em solos tropicais altamente intemperizados não está satisfatoriamente esclarecida (Novais \& Smyth, 1999).

O objetivo deste trabalho foi avaliar os teores totais de $\mathrm{P}$, assim como sua disponibilidade e retenção em Latossolos sob pastagens do Cerrado, relacionando-os com as características mineralógicas, granulométricas e químicas dos solos. 


\section{MATERIAL E MÉTODOS}

\section{Sub-regiões e amostragem}

As amostras foram coletadas em Latossolos, sob pastagens de diferentes graus de fertilidade química, em duas regiões do bioma Cerrado: entre as cidades de Goiânia, GO, e Barra do Garças, MT, próximo às rodovias BR-060 e BR-154, e nos municípios de Unaí e Paracatu, noroeste de Minas Gerais.

As amostras de solos (32 no total) foram retiradas na camada $0-20 \mathrm{~cm}$, com auxílio de trado tipo holandês (material inoxidável), sendo realizadas duas tradagens por ponto de coleta. Todos os pontos foram georreferenciados. Após a coleta, as amostras foram empacotadas e identificadas e, em seguida, enviadas ao laboratório de solos da Embrapa Cerrados, onde foram secas e passadas em peneira $2 \mathrm{~mm}$.

\section{Análises de solos}

As análises fisicas e químicas foram realizadas de acordo com Embrapa (1997). Determinaram-se as seguintes características: granulometria; $\mathrm{pH}$ em água; $\mathrm{P}$ e K disponíveis, extraídos com solução Mehlich-1; $\mathrm{Al}$, Ca e Mg trocáveis, extraídos com KCl 1,0 mol L-1; $\mathrm{H}+\mathrm{Al}$, extraído com solução do acetato de cálcio $0,5 \mathrm{~mol} \mathrm{~L}^{-1}, \mathrm{pH} 7,0$. A partir destes dados, foram calculadas a soma de bases trocáveis (SB), a capacidade de troca catiônica (CTC) e a saturação por bases (V). O C total foi determinado por combustão a seco (CHNS/O), em analisador elementar, modelo Perkin Elmer, PE-2400 Séries II. O P também foi determinado em resina trocadora de ânions de acordo com Raij et al. (2001).

Adicionalmente, em subamostras de cada ponto, foi realizado ataque sulfúrico para determinação dos teores totais de $\mathrm{Fe}_{2} \mathrm{O}_{3}, \mathrm{Al}_{2} \mathrm{O}_{3}, \mathrm{P}_{2} \mathrm{O}_{5}$ e $\mathrm{SiO}_{2}$ (Embrapa, 1997). Dissoluções seletivas dos compostos de $\mathrm{Al}$ e de Fe foram efetuadas por meio de extrações com oxalato ácido de amônia (Tamm) e citrato-ditionito-bicarbonato (CBD). A extração pelo Tamm foi feita agitando-se $4 \mathrm{~g}$ de solo em $40 \mathrm{~mL}$ de reagente com 10,92 $\mathrm{g} \mathrm{L}^{-1}$ de ácido oxálico $\left(\mathrm{C}_{2} \mathrm{O}_{4} \mathrm{H}_{2} 2 \mathrm{H}_{2} \mathrm{O}\right)+$ oxalato de amônia $\left(\mathrm{C}_{2} \mathrm{O}_{4}\left(\mathrm{NH}_{4}\right)_{2}, \mathrm{H}_{2} \mathrm{O}\right)$, no escuro, a $20^{\circ} \mathrm{C}$, por $4 \mathrm{~h}$ (McKeague \& Day, 1966). Para a extração CDB (Mehra \& Jackson, 1960), agitou-se 1,0 g de solo em $50 \mathrm{~mL}$ de reativo com tricitrato de sódio $\left(\mathrm{Na}_{3} \mathrm{C}_{6} \mathrm{H}_{5} \mathrm{O}_{7}\right.$, $\left.2 \mathrm{H}_{2} \mathrm{O}\right)+$ hidrogenocarbonato de sódio $\left(\mathrm{NaHCO}_{3}\right)$, por $30 \mathrm{~min}$, em banho-maria, a $80{ }^{\circ} \mathrm{C}$. Após, adicionouse 1,0 g de ditionito $\left(\mathrm{Na}_{2} \mathrm{~S}_{2} \mathrm{O}_{4}\right)$ e colocou-se em banhomaria por mais $15 \mathrm{~min}, \mathrm{a} 80^{\circ} \mathrm{C}$. Todos os resultados foram obtidos mediante utilização de aparelho ICPAES (Inductively Coupled Plasma Atomic Emission Spectroscopy). O Fe e o $\mathrm{Al}$ extraídos pelo oxalato foram denominados $\mathrm{Fe}_{0}$ e $\mathrm{Al}_{0}$, e os extraídos pelo CBD, $\mathrm{Fe}_{\mathrm{d}}$ e $\mathrm{Al}_{\mathrm{d}}$.

O P remanescente (Prem) foi determinado em solução resultante da agitação de 5,0 g de solo, por uma hora, em solução preparada com $50 \mathrm{~mL}$ de $\mathrm{CaCl}_{2}$ $0,01 \mathrm{~mol} \mathrm{~L}^{-1}$, que continha $60 \mathrm{mg} \mathrm{L}^{-1} \mathrm{de}$ P. Antes da determinação, a solução de equilíbrio foi centrifugada por 5 min e, em seguida, filtrada (Alvarez V. et al., 2000).

\section{Análise estatística}

Os resultados experimentais foram submetidos à análise de componentes principais (ACP) por meio do programa ADE-4 (Thioulouse et al., 1997), utilizando uma matriz dos 32 pontos $\times 28$ variáveis fisicas e químicas dos solos, a fim de analisar as inter-relações entre as variáveis relativas à disponibilidade e retenção do P com as demais. A ACP baseia-se na transformação linear de "n" variáveis originais em "n" variáveis produzidas pelo sistema. Os eixos $x$ e $y$, denominados componentes principais, podem ser vistos como "supervariáveis", construídas pela combinação da correlação entre as variáveis, estes são extraídos em ordem decrescente de importância de acordo com sua contribuição para a variação total dos dados. Os componentes principais, dispostos num espaço de duas dimensões, representam variabilidade suficiente que possa indicar algum padrão a ser interpretado. Autovetor é o valor que representa o peso de cada variável em cada componente (eixos) e funciona como coeficiente de correlação que varia de $-1 \mathrm{a}+1$. As variáveis com elevado autovetor no primeiro eixo tendem a ter autovetor inferior no segundo eixo (Gomes et al., 2004; Tino, 2005). Os coeficientes de correlação de Pearson, utilizados como medida de dependência entre as variáveis estudadas, foram estimados por meio do aplicativo SAS (SAS, 1999).

\section{RESULTADOS E DISCUSSÃO}

\section{Caracterização dos Latossolos}

As médias, desvio-padrão, mínimos e máximos dos resultados das análises químicas, granulométricas e mineralógicas estão apresentados no quadro 1.

Os valores de $\mathrm{pH}_{\mathrm{H} 2 \mathrm{O}}$ encontrados nos solos variaram de 4,9 a 6,5, segundo Raij et al. (1996), são considerados de baixo a alto. Apresentam soma de bases entre 0,6 a $4,4 \mathrm{cmol}_{\mathrm{c}} \mathrm{kg}^{-1}$, teores de $\mathrm{Al}$ trocável $\left(\mathrm{Al}^{3+}\right)$, variando de 0,0 a $1,4 \mathrm{cmol}_{\mathrm{c}} \mathrm{kg}^{-1}$, e saturação por bases, de 8,3 a $58,7 \%$. Os teores de $\mathrm{C}$ total encontrados nos 32 Latossolos são baixos, com média de $13,3 \mathrm{~g} \mathrm{~kg}^{-1}$. Tais características comprovam a grande variabilidade da fertilidade química dos Latossolos do Cerrado (Quadro 1).

Os teores de argila variaram de 18 a $72 \%$, os de silte, areia fina e areia grossa, de 0 a 14, 5 a 61 e 3 a $44 \%$, respectivamente (Quadro 1), valores próximos aos encontrados por Macêdo (1996). 
Quadro 1. Média, desvio-padrão (DP), valor mínimo e máximo das variáveis químicas, físicas e mineralógicas de Latossolos sob pastagem do Cerrado

\begin{tabular}{|c|c|c|c|c|}
\hline Variável $^{(1)}$ & Média & DP & Mínimo & Máximo \\
\hline Argila (\%) & 40,4 & 15,3 & 18,0 & 72,0 \\
\hline Silte (\%) & 5,8 & 3,9 & 0,0 & 14,0 \\
\hline Areia fina $(\%)$ & 32,5 & 11,7 & 5,0 & 61,0 \\
\hline Areia grossa (\%) & 21,3 & 11,7 & 3,0 & 44,0 \\
\hline $\mathrm{pH}$ & 5,5 & 0,4 & 4,9 & 6,5 \\
\hline $\mathrm{H}+\mathrm{Al}\left(\mathrm{cmol}_{\mathrm{c}} \mathrm{kg}^{-1}\right)$ & 4,0 & 1,1 & 2,2 & 6,4 \\
\hline $\mathrm{Al}\left(\mathrm{cmol}_{\mathrm{c}} \mathrm{kg}^{-1}\right)$ & 0,5 & 0,4 & 0,0 & 1,4 \\
\hline $\mathrm{K}\left(\mathrm{cmol}_{\mathrm{c}} \mathrm{kg}^{-1}\right)$ & 0,1 & 0,1 & 0,0 & 0,7 \\
\hline $\mathrm{Ca}\left(\mathrm{cmol}_{\mathrm{c}} \mathrm{kg}^{-1}\right)$ & 1,1 & 1,0 & 0,0 & 3,0 \\
\hline $\mathrm{Mg}\left(\mathrm{cmol}_{\mathrm{c}} \mathrm{kg}^{-1}\right)$ & 0,8 & 0,4 & 0,2 & 1,7 \\
\hline $\mathrm{CTC}\left(\mathrm{cmol}_{\mathrm{c}} \mathrm{kg}^{-1}\right)$ & 6,1 & 1,1 & 3,5 & 7,7 \\
\hline $\mathrm{SB}\left(\mathrm{cmol}_{\mathrm{c}} \mathrm{kg}^{-1}\right)$ & 2,1 & 1,2 & 0,6 & 4,4 \\
\hline $\mathrm{V}(\%)$ & 33,1 & 16,6 & 8,3 & 58,7 \\
\hline $\operatorname{Pmeh}\left(\mathrm{mg} \mathrm{kg}^{-1}\right)$ & 1,0 & 0,4 & 0,1 & 1,7 \\
\hline Pres $\left(\mathrm{mg} \mathrm{kg}^{-1}\right)$ & 7,9 & 1,4 & 5,2 & 11,1 \\
\hline Prem $\left(\mathrm{mg} \mathrm{L}^{-1}\right)$ & 23,6 & 10,7 & 5,2 & 40,8 \\
\hline $\mathrm{C}_{\text {total }}\left(\mathrm{g} \mathrm{kg}^{-1}\right)$ & 13,3 & 5,4 & 3,3 & 22,8 \\
\hline $\mathrm{Al}_{2} \mathrm{O}_{3}\left(\mathrm{~g} \mathrm{~kg}^{-1}\right)$ & 154,6 & 52,6 & 56,0 & 239,0 \\
\hline $\mathrm{Fe}_{2} \mathrm{O}_{3}\left(\mathrm{~g} \mathrm{~kg}^{-1}\right)$ & 70,2 & 34,3 & 17,0 & 140,0 \\
\hline $\mathrm{P}_{2} \mathrm{O}_{5}\left(\mathrm{~g} \mathrm{~kg}^{-1}\right)$ & 0,9 & 0,4 & 0,2 & 2,6 \\
\hline $\mathrm{Ct}\left(\mathrm{g} \mathrm{kg}^{-1}\right)$ & 197,4 & 76,3 & 98,8 & 345,8 \\
\hline $\mathrm{Gb}\left(\mathrm{g} \mathrm{kg}^{-1}\right)$ & 117,2 & 73,6 & 5,7 & 277,3 \\
\hline Go $\left(\mathrm{g} \mathrm{kg}^{-1}\right)$ & 47,6 & 35,8 & 5,7 & 135,7 \\
\hline $\mathrm{Hm}\left(\mathrm{g} \mathrm{kg}^{-1}\right)$ & 27,3 & 29,1 & 0,0 & 105,6 \\
\hline Alo $\left(\mathrm{g} \mathrm{kg}^{-1}\right)$ & 1,6 & 0,8 & 0,6 & 3,1 \\
\hline Feo $\left(\mathrm{g} \mathrm{kg}^{-1}\right)$ & 0,9 & 0,4 & 0,2 & 1,7 \\
\hline Ald $\left(\mathrm{g} \mathrm{kg}^{-1}\right)$ & 2,8 & 1,5 & 0,8 & 6,9 \\
\hline Fed $\left(\mathrm{g} \mathrm{kg}^{-1}\right)$ & 25,8 & 21,2 & 4,7 & 92,0 \\
\hline
\end{tabular}

(1) CTC: capacidade de troca catiônica; SB: soma de bases; Pmeh: fósforo Mehlich; Pres: fósforo resina; Prem: fósforo remanescente; $\mathrm{Al}_{2} \mathrm{O}_{3}, \mathrm{Fe}_{2} \mathrm{O}_{3}$ e $\mathrm{P}_{2} \mathrm{O}_{5}$ : alumínio, ferro e fósforo totais; $\mathrm{Ct}$, $\mathrm{Gb}$, Go e Hm: caulinita, gibbsita, goethita e hematita; Alo e Feo: óxidos de alumínio e ferro extraído por oxalato ácido de amônia; Ald e Fed: óxidos de alumínio e ferro extraído por citrato-ditionito-bicarbonato.

Na fração argila, constatou-se predominância de caulinita (Ct) e gibbsita (Gb) e, em quantidades menores, goethita (Go) e hematita $(\mathrm{Hm})$, resultado similar ao encontrado por Alves \& Lavorenti (2004). As dissoluções seletivas extraídas com Tamm e CBD indicam que a maior parte dos óxidos de $\mathrm{Fe}$ e de $\mathrm{Al}$ está na forma bem cristalizada, condizente com a condição de solos muito intemperizados. A fração cristalizada de $\mathrm{Al}$ é representada principalmente por $\mathrm{Ct}$ e $\mathrm{Gb}$, e a de $\mathrm{Fe}$ por Go e Hm, elucidando a presença destes minerais.

\section{Teores de fósforo nas pastagens}

$\mathrm{O}$ teor de $\mathrm{P}$ total $\left(\mathrm{P}_{2} \mathrm{O}_{5}\right)$ variou de 0,2 a $2,6 \mathrm{~g} \mathrm{~kg}^{-1}$, com média de $0,9 \mathrm{~g} \mathrm{~kg}^{-1}$ (Quadro 1). A média e a distribuição dos teores de $\mathrm{P}$ total nas pastagens (Figura 1) foram semelhantes às de Marques et al. (2004) para o Cerrado (média: 0,8 $\mathrm{g} \mathrm{kg}^{-1}$; amplitude: 0,14 a $\left.4,37 \mathrm{~g} \mathrm{~kg}^{-1}\right)$.

Observou-se que os teores de $\mathrm{P}$ disponível Mehlich (Pmeh) apresentaram amplitude baixa (Figura 1), variando de 0,1 a $1,7 \mathrm{mg} \mathrm{kg}^{-1}$ e média de $1,0 \mathrm{mg} \mathrm{kg}^{-1}$ (Quadro 1). Este valor de P disponível representa apenas $0,25 \%$, em média, do $\mathrm{P}$ total dos solos sob pastagens. Os teores de $\mathrm{P}$ disponível resina (Pres) variaram de 5,2 a $11,1 \mathrm{mg} \mathrm{kg}^{-1}$ com média de $7,9 \mathrm{mg} \mathrm{kg}^{-1}$ (Quadro 1), tendo uma amplitude maior que o Pmeh. Os teores de Pres são classificados como muito baixos ( 4 solos, $12,5 \%$ ), baixos ( 25 solos, $78,1 \%$ ) e adequados ( 3 solos, 9,4 \%), segundo Souza \& Lobato (2004). Os baixos valores de P disponível encontrados reforçam a idéia descrita por Souza et al. (1998) e Macedo (2004) de que o P é um dos fatores restritivos ao desenvolvimento adequado das pastagens, tornando-se fator limitante para o desenvolvimento da pecuária na região do Cerrado.

Os teores de $\mathrm{P}$ remanescente (Prem) variaram de 5,2 a 40,8 $\mathrm{mg} \mathrm{L}^{-1}$ (Quadro 1). De acordo com Alvarez V. et al. (2000), todos os Latossolos analisados neste estudo apresentam alta retenção de P (Figura 1). A amplitude dos valores de Prem pode estar relacionada com a variabilidade das características físico-químicas, granulométricas e mineralógicas desses solos.

\section{Efeitos das características dos solos na retenção e disponibilidade de $\mathbf{P}$}

Os valores de Pmeh não se correlacionaram com os demais atributos avaliados, o que ocorreu apenas com o $\mathrm{Al}$ trocável $\left(\mathrm{r}=0,48^{* *}\right)$. A correlação positiva com o Al trocável pode ser atribuída ao fato dos solos com predominância de $\mathrm{Ct}$ em relação a $\mathrm{Gb}$ apresentarem teores mais elevados desse elemento (Vendrame et al., 2005). Além disso, os solos ricos em Ct retêm menos P (Frossard et al., 1995; Rolim Neto et al., 2004).

A ausência de correlação do Pmeh com os demais atributos avaliados pode estar relacionada com o fato de o teor de $\mathrm{P}$ disponível no solo depender, principalmente, do manejo, do sistema de produção e da adubação. O extrator Mehlich-1, uma solução ácida, também tem efeito importante sobre o $\mathrm{pH}$ da suspensão no processo de extração e, segundo Dubus \& Becquer (2001), a redução do pH tem implicações tanto no grau de saturação dos íons de ortofosfatos, aumentando as formas $\mathrm{H}_{2} \mathrm{PO}_{4}{ }^{-}$em relação às de $\mathrm{HPO}_{4}{ }^{-2}$, quanto nas mudanças de cargas da superfície dos óxidos, aumentando as cargas eletropositivas. O conjunto dessas modificações tem efeitos opostos, podendo alterar algumas relações entre as formas do P em solução e as características dos solos. 

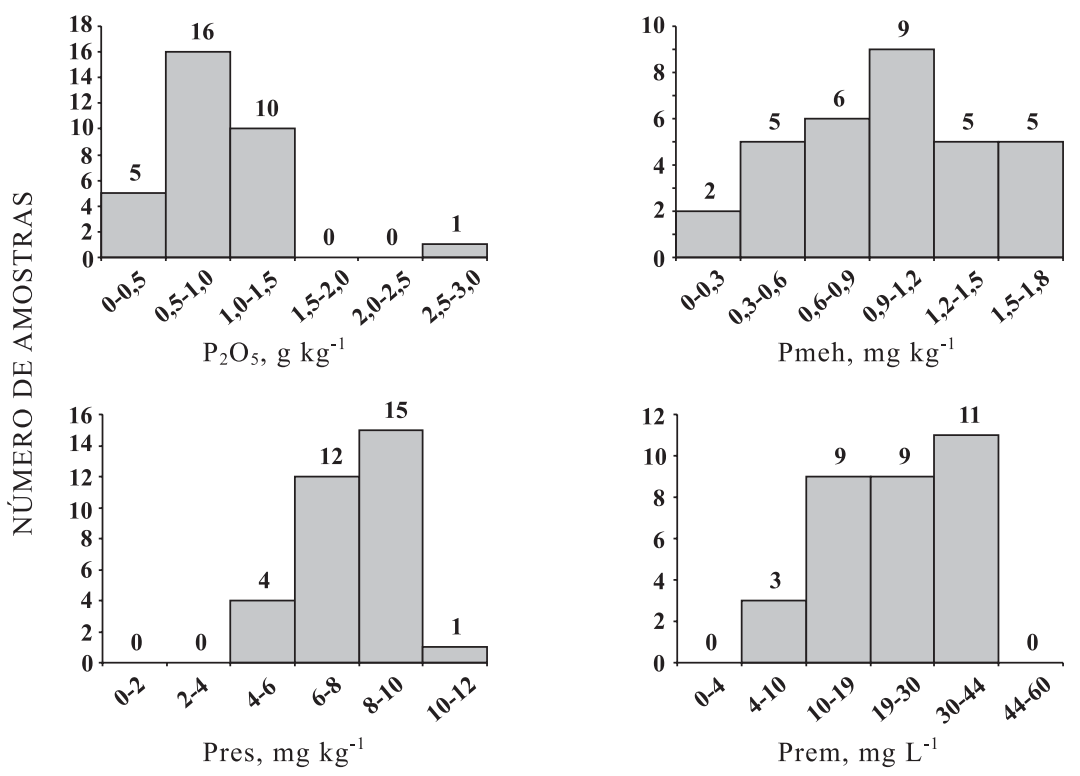

Figura 1. Distribuição de freqüência dos teores de fósforo total $\left(\mathrm{P}_{2} \mathrm{O}_{5}\right)$, fósforo Mehlich (Pmeh), fósforo resina (Pres) e fósforo remanescente (Prem) em Latossolos sob pastagens no Cerrado.

O Pres correlacionou-se com o teor de argila, C total, $\mathrm{Fe}_{\mathrm{o}}$ e $\mathrm{SB}\left(\mathrm{r}=0,41^{*}, 0,44^{*}, 0,41^{*}\right.$, e $0,42^{*}$, respectivamente). Nos Latossolos, o C orgânico (C) está concentrado mais nas frações de argila e silte (Freitas et al., 2000) e o aumento no teor de C está significativamente relacionado com o teor de argila (Zinn et al., 2005). Conseqüentemente, a ação bloqueadora da matéria orgânica sobre os sítios de adsorção dos ânions (Afif et al., 1995) pode explicar a correlação do $\mathrm{C}$ total com o Pres nos solos argiloso.

Os teores de $\mathrm{P}$ disponível determinados por Mehlich-1 e Resina não se correlacionaram, o que deve ser reflexo das diferenças na capacidade extratora de cada método. De acordo com Novais \& Smyth (1999), o método da resina reflete melhor o fator quantidade de $\mathrm{P}$ no solo, enquanto o Mehlich-1 representa fração do fator quantidade, influenciada pelo fator capacidadetampão de $\mathrm{P}$ do solo.

A sorção do $\mathrm{P}$ pelos minerais do solo vem sendo estudada desde a década de 50 (Frossard et al., 1995). $\mathrm{A} \mathrm{Gb}, \mathrm{a}$ Go e a Hm apresentaram correlações negativas com o Prem $\left(r=-0,64^{* *},-0,64^{* *}\right.$ e $-0,43^{*}$ respectivamente). Já a Ct, não exerceu influência sobre o Prem $(\mathrm{r}=-0,17)$, mostrando a baixa participação desta na adsorção de P nos Latossolos do Cerrado. Estes resultados estão de acordo com os obtidos por Fontes \& Weed (1996) em um estudo sobre a adsorção de P em argilas de Latossolos brasileiros. Estes autores demonstraram, por meio de uma análise de regressão múltipla, que os óxidos de $\mathrm{Al}$ amorfos, $\mathrm{Gb}$, Go e Hm, explicaram mais de $95 \%$ da variação na adsorção máxima de fosfato.

Diferentemente do encontrado na literatura (Frossard et al., 1995; Fontes \& Weed, 1996), observou- se, neste estudo, que a Gb foi tão efetiva quanto a Go na adsorção de P. A influência da Gb sobre o Prem é devida às quantidades de $\mathrm{Gb}$, que são maiores que as de óxidos de $\mathrm{Fe}$, evidenciando a importância dos teores e da superfície específica dos óxidos de $\mathrm{Fe}$ e $\mathrm{Al}$ (Go e $\mathrm{Gb})$ no aumento da adsorção de fosfato.

Os solos desenvolvidos sobre basaltos são, em sua maioria, ricos em $\mathrm{Fe}$, o que explica o papel importante dos óxidos de Fe nesses solos. Alves \& Lavorenti (2004), em Latossolos do Estado de São Paulo, demonstraram, em análise de regressão, que Hm e Go tiveram efeitos significativos sobre o Prem. Nos solos estudados, a $\mathrm{Hm}$, em relação a Go, teve baixa correlação, graças às variações em suas propriedades intrínsecas, tais como o grau de cristalinidade, substituição do $\mathrm{Al}$, superfície específica e faces expostas para adsorção, com o Prem (Alves \& Lavorenti, 2004).

A ausência de influência da Ct sobre o Prem demonstra sua baixa participação na adsorção de $\mathrm{P}$ em relação a Gb, Go e Hm, como observado por Ker (1995), Rolim Neto et al. (2004) e Moreira et al. (2006). A baixa capacidade de adsorção da MOS, em relação aos óxidos de $\mathrm{Fe}$ e de $\mathrm{Al}$, mais eficazes e ativos na adsorção de $\mathrm{P}$, pode ser relacionada principalmente com a carga de superfície dos minerais a $\mathrm{pH}$ do solo. Nesta faixa de $\mathrm{pH}(4,9-6,5)$, de acordo com Barrow (1985), os óxidos de $\mathrm{Fe}$ e de $\mathrm{Al}$ apresentam cargas positivas, atraindo o ânion ortofosfato, enquanto, a MOS tem cargas negativas, repulsando o ânion ortofosfato.

Os óxidos de $\mathrm{Fe}$ e de Al livres e amorfos apresentaram correlação negativa com o Prem $\left(r=-0,66^{* *}\right.$, $-0,77^{* *},-0,70^{* *}$ e $-0,80^{* *}$ respectivamente). Porém, os óxidos de $\mathrm{Fe}$ e $\mathrm{Al}$ amorfos $\left(\mathrm{Fe}_{0}\right.$ e $\left.\mathrm{Al}_{0}\right)$, extraídos com 
oxalato ácido de amônia (Tamm), apresentaram maior correlação negativa que os óxidos de $\mathrm{Fe}$ e de $\mathrm{Al}$ livres $\left(\mathrm{Fe}_{\mathrm{d}}\right.$ e $\left.\mathrm{Al}_{\mathrm{d}}\right)$ extraídos com citrato-ditionito-bicarbonato (CBD). As correlações com os óxidos livres (superfície específica menor) evidenciaram a influência destes na adsorção de P. Estes resultados também foram encontrados por Borggaard (1983), confirmando que o óxido de $\mathrm{Fe}$ amorfo tem efeito maior que o livre, pois a capacidade de adsorção dos óxidos está diretamente ligada à superfície específica que diminui com o aumento da cristalinidade.

A textura do solo teve influência sobre o Prem, uma vez que todas as frações tiveram correlação com ele, com oposição entre as partículas finas (fração argila e silte), com correlação negativa $\left(r=-0,82^{* *}\right.$ e $-0,69 * *$ respectivamente), e as partículas grossas (fração areia fina e grossa), com correlação positiva $\left(\mathrm{r}=0,60^{* *} \mathrm{e}\right.$ $\left.0,71^{* *}\right)$. Tal influência da textura é devida à presença de óxidos de $\mathrm{Fe}$ e de $\mathrm{Al}$ (gibsita, goethita, óxidos de $\mathrm{Fe}$ e Al livres e amorfos) na fração argila.

Observou-se correlação negativa entre o Prem e o $\mathrm{C}$ total $\left(\mathrm{r}=-0,68^{* *}\right)$. O C orgânico $(\mathrm{C})$ pode influir na adsorção de $\mathrm{P}$, positivamente (Brennan et al., 1994), negativamente (Parfitt, 1978), ou ainda não ter efeito (Borggaard et al., 1990). Mais recentemente, Zinn et al. (2005) observaram em solos de Cerrado que o estoque de $\mathrm{C}$ aumenta linearmente com o conteúdo de argila e de silte do solo. Neste trabalho, observou-se que a fração fina é positivamente correlacionada com o C total, $\left(\mathrm{r}=0,747^{* *}\right.$ e $0,658^{* *}$ para argila e silte, respectivamente), demonstrando que o $\mathrm{C}$ total não aumenta a adsorção de $\mathrm{P}$, mas este aumenta com a riqueza dos minerais que retêm o $\mathrm{P}$, que, por sua vez, está intimamente ligado à fração fina do solo.

Não foi observada correlação entre $\mathrm{pH}$ e Prem $(\mathrm{r}=$ $-0,20)$. Correlações positivas, negativas ou nãosignificativas também foram encontradas por vários autores, demonstrando efeitos complexos e, muitas vezes, antagônicos do $\mathrm{pH}$ sobre a sorção de $\mathrm{P}$ (Barrow, 1985; Geelhoed et al., 1999; Sato \& Comerford, 2005).

A análise de componentes principais (ACP) foi utilizada para reduzir as dimensões dos dados e, conseqüentemente, facilitar a análise por meio do gráfico do círculo de correlações (Thioulouse et al., 1997; Herlihy \& McCarthy, 2006). A ACP realizada sobre o conjunto de dados físicos e químicos, granulométricos e mineralógicos dos solos revelou que os dois primeiros eixos explicaram $56 \%$ da variabilidade total dos dados, sendo $38 \%\left(1^{\circ} \mathrm{E}\right)$ e $18 \%$ $\left(2^{\circ} \mathrm{E}\right)$ (Figura 2). O eixo 1 foi influenciado especialmente pela mineralogia e pela textura dos Latossolos, em que a fração grosseira (areia grossa e areia fina) e o Prem apresentaram autovetores positivos. A fração mais fina (silte e argila), os componentes mineralógicos (Ct, Gb, Go, Hm e os óxidos de $\mathrm{Fe}$ e $\mathrm{Al} \mathrm{livres} \mathrm{e} \mathrm{amorfos),}$ os teores totais de $\mathrm{Fe}, \mathrm{Al}$ e $\mathrm{P}\left(\mathrm{Fe}_{2} \mathrm{O}_{3}, \mathrm{Al}_{2} \mathrm{O}_{3}\right.$ e $\left.\mathrm{P}_{2} \mathrm{O}_{5}\right)$, CTC, C total e o Pres apresentaram autovetores negativos. $\mathrm{O}$ eixo 2 foi influenciado pelas variáveis oriundas da análise química do solo, tendo sido observada separação entre os elementos básicos e ácidos. Dessa forma, $\mathrm{Al}^{3+}$ e $\mathrm{H}+\mathrm{Al}$ apresentaram autovetores negativos, assim como o Pmeh, enquanto $\mathrm{V}, \mathrm{pH}, \mathrm{Mg}, \mathrm{SB}$ e Ca apresentaram autovetores positivos (Figura 2).

Finalmente, observou-se que os autovetores do Prem e os que representam a mineralogia do solo apresentaram comportamento antagônico, evidenciando a capacidade dos óxidos de $\mathrm{Fe}$ e $\mathrm{Al}$ em reter o P. Neste mesmo sentido, observou-se que estes minerais da fração argila exercem influência sobre os teores de $\mathrm{P}$ total, considerando a grande quantidade de $\mathrm{P}$ adsorvida nestes minerais. O Pres está relacionado com o lado negativo do eixo 1; entretanto, com uma contribuição fraca, graças aos efeitos antagonistas dos óxidos de $\mathrm{Fe}$ e $\mathrm{Al}$ na sorção e da matéria orgânica na disponibilidade de P. Ao contrário do Pres, o Pmeh não apresenta correlações com a mineralogia e granulometria, evidenciando que ele representa melhor o fator-capacidade de $\mathrm{P}$ do solo do que a disponibilidade.

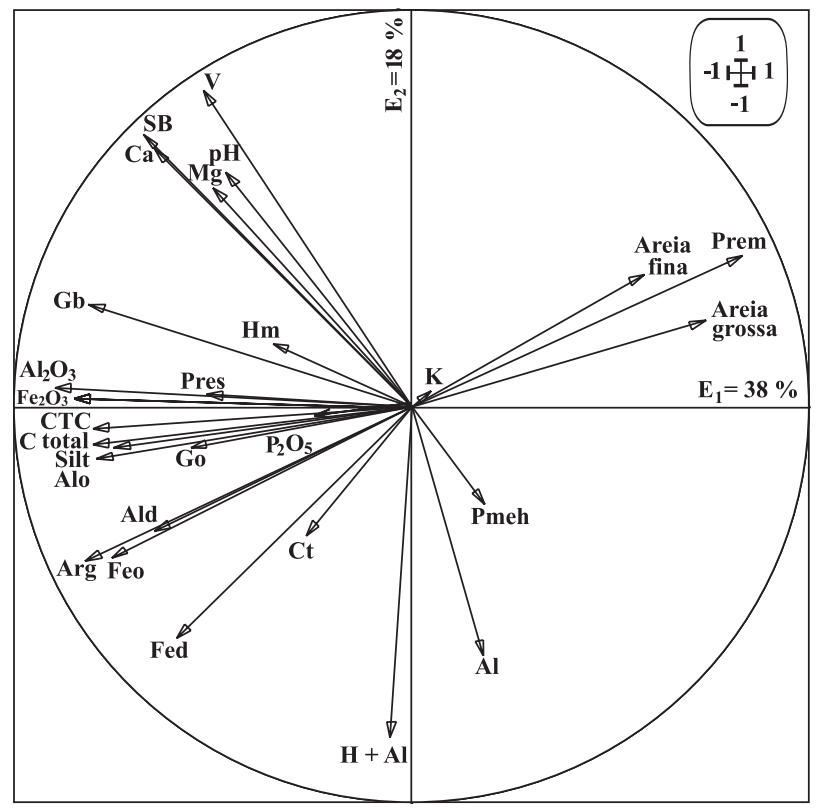

Figura 2. Círculo de correlações entre as variáveis Argila, Silte, Areia grossa, Areia fina, $\mathrm{pH}, \mathrm{H}+\mathrm{Al}$, $\mathrm{Al}^{3+}, \mathrm{K}, \mathrm{Ca}, \mathrm{Mg}, \mathrm{CTC}$ (capacidade de troca catiônica), SB (soma de bases), V (saturação por bases), Pmeh (fósforo Mehlich), Pres (fósforo resina), Prem (fósforo remanescente), $\mathrm{C}$ total (Carbono), $\mathrm{Al}_{2} \mathrm{O}_{3}$ (alumínio total), $\mathrm{Fe}_{2} \mathrm{O}_{3}$ (ferro total) e $\mathrm{P}_{2} \mathrm{O}_{5}$ (fósforo total), Caulinita (Ct), Gibbsita (Gb), Goethita (Go), Hematita (Hm), Alo (óxidos de alumínio amorfos), Feo (óxidos de ferro amorfos), Ald (óxidos de alumínio livres), Fed (óxidos de ferro livres) de Latossolos sob pastagem do Cerrado. 


\section{CONCLUSÕES}

1. O P remanescente mostrou-se relacionado com a mineralogia da fração argila dos Latossolos do Cerrado, destacando-se a gibbsita, abundante nestes solos, e os óxidos de Fe e de $\mathrm{Al}$ livres e amorfos.

2. O P disponível resina relacionou-se com as características mineralógicas e granulométricas, assim como com os teores de matéria orgânica.

3. O P Mehlich não se correlacionou com o P resina, tampouco com as características mineralógicas e granulométricas dos solos.

\section{AGRADECIMENTOS}

Ao laboratório de solos da Universidade Estadual de Londrina (UEL) e aos doutorandos Alfredo Richart e Cristine Elizabeth Alvarenga Carneiro. Ao Institut de Recherche pour le Développement (IRD), pelo apoio financeiro. Aos doutores Angel Rosales e Robélio Leandro Marchão, pelo auxílio nas análises estatísticas, correções e sugestões.

\section{LITERATURA CITADA}

AFIF, E.; BARRÓN, V. \& TORRENT, J. Organic matter delays but does not prevent phosphate sorption by Cerrado soils from Brazil. Soil Sci., 159:207-211, 1995.

ALVAREZ V., V.H.; NOVAIS, R.F.; DIAS, L.E. \& OLIVEIRA, J.A. Determinação e uso do fósforo remanescente. B. Inf. SBCS, 25:27-33, 2000.

ALVES, M.E. \& LAVORENTI, A. Remaining phosphorus and sodium fluoride $\mathrm{pH}$ in soils with different clay contents and clay mineralogies. Pesq. Agropec. Bras., 39:241-246, 2004.

BAHIA FILHO, A.F.C. Índices de disponibilidade de fósforo em Latossolos do planalto central com diferentes características texturais e mineralógicas. Viçosa, MG, Universidade Federal de Viçosa, 1982.179p. (Tese de Doutorado)

BARROW, N.J. Reaction of anions and cations with variablecharge soils. Adv. Agron., 38:183-230, 1985.

BORGGAARD, O.K. The influence of iron oxides on phosphate adsorption by soil. J. Soil Sci., 34:333-341, 1983.

BORGGAARD, O.K.; JORGENSEN, S.S.; MOBERG, J.P. \& RABEN-LAGE, B. Influence of organic matter on phosphate adsorption by aluminum and iron oxides in sandy soils. J. Soil Sci., 41:443-449, 1990.

BRENNAN, R.F.; BOLLAND M.D.A.; JEFFERY, R.C. \& ALLEN, D.G. Phosphorus adsorption by a range of western Australian soils related to soil properties. Comm. Soil Sci. Plant Anal., 25:2785-2795, 1994.
BROSSARD, M. \& BARCELLOS, A.O. Conversão do cerrado em pastagens cultivadas e funcionamento de latossolos. Cader. Ci. Tecnol., 22:153-168, 2005.

DUBUS, I.G. \& BECQUER, T. Phosphorus sorption and desorption in oxide-rich ferasols of New Caledonia. Aust. J. Soil Res., 39:403-414, 2001.

EMPRESA BRASILEIRA DE PEQUISA AGROPECUÁRIA EMBRAPA. Centro Nacional de Pesquisa de Solos. Manual de métodos de análise de solo. 2.ed. Rio de Janeiro, 1997. 212p.

FONTES, M.P.F. \& WEED, S.B. Phosphate adsorption by clays from Brazilian Oxisols: Relationships with specific surface area and mineralogy. Geoderma, 72:37-51, 1996.

FREITAS, P.L.; BLANCANEAUX, P.; GAVINELLI, E.; LARRÉLARROUY, M.C. \& FELLER, C. Nível e natureza do estoque orgânico de latossolos sob diferentes sistemas de uso e manejo. Pesq. Agropec. Bras., 35:157-170, 2000.

FROSSARD, E.; BROSSARD, M.; HEDLEY, M.J. \& METHERELL, A. Reactions controlling the cycling of $\mathrm{P}$ in soils. In: TIESSEN, H., ed. Phosphorus in the global environment, transfers, cycles and management. Scope 54. New York, John Wiley \& Sons, 1995. p.107-137.

GEELHOED, J.S.; VANRIEMSDIJK, W.H. \& FINDENEGG, G.R. Simulation of the effect of citrate exudation from roots on the plant availability of phosphate adsorbed on goethite. Eur. J. Soil Sci., 50:379-390, 1999.

GOMES, J.B.V.; CURI, N.; MOTTA, P.E.F.; KER, J.C.; MARQUES, J.J.G.S. M. \& SCHULZE, D.G. Análise de componentes principais de atributos físicos, químicos e mineralógicos de solos do bioma cerrado. R. Bras. Ci. Solo, 28:137-153, 2004.

HERLIHY, M. \& McCARTHY, J. Association of soil-test phosphorus with phosphorus fractions and adsorption characteristics. Nutr. Cycl. Agroecosyst., 75:79-90, 2006.

KER, J.C. Mineralogia, sorção e dessorção de fosfato, magnetização e elementos traços de latossolos do Brasil. Viçosa, MG, Universidade Federal de Viçosa, 1995. 181p. (Tese de Doutorado)

MACÊDO, J. Os solos da região dos Cerrados. In: ALVAREZ V, V.H.; FONTES, L.E.F. \& FONTES, M.P.F. Os solos nos grandes domínios morfoclimáticos do Brasil e desenvolvimento sustentado. Viçosa, MG, Sociedade Brasileira de Ciência do Solo, 1996. p.135-167.

MACEDO, M.C.M. Análise comparativa de recomendações de adubação em pastagens. In: SIMPÓSIO SOBRE O MANEJO DA PASTAGEM, 21., Piracicaba, 2004. Fertilidade do solo para pastagens produtivas. Piracicaba, FEALQ, 2004. p. 317-355.

MARQUES, J.J.; SCHULZE, D.G.; CURI, N. \& MERTZMAN, S.A. Major element geochemistry and geomorphic relationships in Brazilian Cerrado soils. Geoderma, 119:179-195, 2004

McKEAGUE, J.A. \& DAY, J.H. Dithionite and oxalateextractable $\mathrm{Fe}$ and $\mathrm{Al}$ as aids in differentiating various classes of soils. Can. J. Soil Sci., 46:13-22, 1966. 
MEHRA, O.P. \& JACKSON, M.L. Iron oxide removal from soils and clays by a dithionite-citrate system buffered with sodium bicarbonate. In: NATIONAL CONFERENCE ON CLAYS AND CLAYS MINERALS, 7., Washington, 1960. Proceedings. New York, Pergamon Press, 1960. p.317. 327.

MOREIRA, L.F.L.M.; MOTA, F.O.B.; CLEMENTE, C.A.; AZEVEDO, B.M. \& BOMFIM, G.V. Adsorção de fósforo em solos do Estado do Ceará. R. Ci. Agron., 37:7-12, 2006.

NOVAIS, R.F. \& SMYTH, T.J. Fósforo em solo e planta em condições tropicais. Viçosa, MG, Universidade Federal de Viçosa, 1999. 399p.

PARFITT, R.L. Anion adsorption by soils and soil materials. Adv. Agron., 30:1-50, 1978.

RAIJ, B.van; CANTARELLA, H.; QUAGGIO, J.A. \& FURLANI, A.M.C. Recomendações de adubação e calagem para o Estado de São Paulo. 2.ed. Campinas, Instituto Agronômico e Fundação IAC, 1996. 255p. (Boletim Técnico, 100).

RAIJ, B. van. \& QUAGGIO, J.A. Determinação de fósforo, cálcio, magnésio e potássio extraídos com resina trocadora de íons. In: RAIJ, B.van.; ANDRADE, J.C.; CANTARELLA, H. \& QUAGGIO, J.A., eds. Análises químicas para avaliação da fertilidade de solos tropicais. Campinas, Instituto Agronômico, 2001. p.189-199.

RESENDE, M.; CURI, N.; RESENDE, S.B. \& CORRÊA, G.F Pedologia: base para distinção de ambientes. Viçosa, MG, NEPUT, 1995. 304p.

ROLIM NETO, F.C.; SCHAEFER, C.E.G.R.; COSTA, L.M.; CORREAA, M.M.; FERNANDES FILHO, E.I. \& IBRAIMO, M.M. Adsorção de fósforo, superfície específica e atributos mineralógicos em solos desenvolvidos de rochas vulcânicas do Alto Paranaíba (MG). R. Bras. Ci. Solo, 28:953-964, 2004.

SANO, E.E.; BARCELLOS, A.O. \& BEZERRA, H.S. Assessing the spatial distribution of cultivated pastures in the Brazilian savanna. Past. Tropic., 22:2-15, 2000.
SAS Institute. SAS/STAT User's guide, Version 8. Cary, 1999.

SATO, S. \& COMERFORD, N.B. Influence of soil $\mathrm{pH}$ on inorganic phosphorus sorption and desorption in a humid brazilian ultisol. R. Bras. Ci. Solo, 29:685-694, 2005.

SOUZA, D.M.G. \& LOBATO, E. Cerrado: correção do solo e adubação. 2.ed. Brasília, Embrapa Informação Tecnológica, 2004. 416p.

SOUZA, D.M.G.; VILELA, L.; LOBATO, E \& SOAREZ, W.V. Uso de gesso, calcário e adubos para pastagens na região do Cerrado. Planaltina, Embrapa-CPAC, 1998. 16p. (Embrapa-CPAC, Circular Técnica, 37)

THIOULOUSE, J.; CHESSEL, D.; DOLÉDEC, S. \& OLIVIER, J.-M. ADE-4: A multivariate analysis and graphical display software. Statistics Comp., 7:75-83, 1997.

TINO, V.F. Utilização da análise de componentes principais para a regulagem de máquinas de injeção plástica. Rio de Janeiro, Universidade Federal do Rio de Janeiro, 2005. (Tese de Mestrado)

TORRENT, J.; SCHWERTMANN, U. \& BARRÓN, V. Phosphate sorption by natural hematites. Eur. J. Soil Sci., 45:45-51, 1994.

VENDRAME, P.R.S.; BRITO, O.R.; GUIMARAES, M.F. \& BECQUER, T. Relação entre características químicas, físicas e mineralógicas de solos sob pastagem no cerrado. In: CONGRESSO BRASILEIRO DE CIÊNCIA DO SOLO, 30.,Recife, 2005. Anais. Viçosa, MG, Sociedade Brasileira de Ciência do Solo, 2005. CD-ROM.

VILELA, L.; MARTHA Jr., G.B.; BARIONI, L.G. \& BARCELLOS, A.O. Adubação na recuperação e na intensificação da produção animal em pastagens. In: SIMPÓSIO SOBRE O MANEJO DA PASTAGEM, 21., Piracicaba, 2004. Fertilidade do solo para pastagens produtivas. Piracicaba, FEALQ, 2004. p.425-472.

ZINN, Y.L.; LAL, R. \& RESCK, D.V.S. Texture and organic carbon relations described by a profile pedotransfer function for Brazilian Cerrado soils. Geoderma,127:168173,2005 . 